\title{
Maternal protein deprivation: sexually dimorphic programming of hypertension in the mouse
}

\author{
Ravi Goyal and Lawrence D Longo
}

Epidemiological data in humans and experiments in laboratory animals have demonstrated that the developmental programming of hypertension may occur as a consequence of dietary manipulations during pregnancy. Surprisingly, there is a scarcity of data regarding the development of hypertension as a consequence of a maternal low-protein diet (MLPD), particularly in the mouse. Furthermore, the role of sex in developmental programming is not well understood. We used FVB/NJ mice, because of their value in genetic/mechanistic analysis, to test the hypothesis that a MLPD during gestation leads to the sexually dimorphic developmental programming of hypertension and related disorders, such as intra-uterine growth restriction (IUGR), type 2 diabetes mellitus and obesity. We administered iso-caloric, normal (control), moderate protein (moderate MLPD) and severe protein (severe MLPD) diets to the mice, beginning 1 week before mating and continuing until the delivery of the pups. From 4 weeks onward, using a non-invasive tail-cuff method, we measured blood pressure and other parameters in the offspring. Our results demonstrate the following: (1) MLPD caused IUGR (low birthweight) in a dose-dependent manner; (2) Female offspring developed severe hypertension, whereas males were affected only moderately; (3) The blood glucose level was elevated only in females from the moderate MLPD group, although their insulin levels remained normal; (4) Rapid catch-up growth was observed in both sexes, with moderate MLPD females and severe MLPD males becoming overweight. Notably, blood leptin levels in the control group were significantly higher in females than in male offspring and were reduced in females from the severe MLPD group. We conclude that an antenatal MLPD during gestation leads to sexually dimorphic programming in mice. Hypertension Research (2013) 36, 29-35; doi:10.1038/hr.2012.129; published online 30 August 2012

Keywords: catch-up growth; fetal programming; leptin; protein malnutrition

\section{INTRODUCTION}

In the western world, essential hypertension along with its associated complications of cardiac, cerebral and renal diseases are leading causes of death. Epidemiological studies in humans ${ }^{1,2}$ and experiments in laboratory animals ${ }^{3-5}$ have suggested that maternal malnutrition during pregnancy may be a major causative factor in the genesis of hypertension. Thus, a factor that has received increasing attention is the idea of 'programming' during fetal life, often occurring as a consequence of maternal stress. ${ }^{6}$ This study presents data demonstrating that a maternal low-protein diet (MLPD) during gestation leads to the sexually dimorphic developmental programming of hypertension and related disorders, such as intra-uterine growth retardation, type 2 diabetes mellitus and obesity.

\section{METHODS}

Experimental animals and tissues

All experimental protocols were in compliance with the Animal Welfare Act, the National Institutes of Health's Guide for the Care and Use of Laboratory Animals and 'The Guiding Principles in the Care and Use of Animals', as approved by the council of the American Physiological Society. The protocols were approved by the Institutional Animal Care and Use Committee of Loma Linda University. The studies were conducted on FVB/NJ mice, a strain selected because their fertilized eggs contain prominent pronuclei that will help in future transgenic studies, and because they have a relatively larger body size compared with other mice strains. The large body size also simplified the measurement of blood pressure using the tail-cuff method at an early age, compared with other mice strains. We obtained mice (approximately 8 weeks of age) from Jackson Laboratories (Bar Harbor, ME, USA) and housed them at the Animal Research Facility, Loma Linda University, under the conditions of $14 \mathrm{~h}$ of light, $10 \mathrm{~h}$ of darkness, an ambient temperature of $20^{\circ} \mathrm{C}$ and relative humidity of 30 to $60 \%$. At 16 weeks of age, the mice were bred by keeping the males and females together for $12 \mathrm{~h}$ (overnight). In the morning, mating was confirmed by the presence of vaginal plugs and was considered 0.5 day post coitum. Pregnancy was confirmed by an increase in weight at the 7 th day post coitum.

\section{Protein deprivation}

Protein-restricted chow was obtained from Newco Distributors (Rancho Cucamonga, CA, USA). Mice dams were divided into three groups: (1) normal (control) protein diet $(18 \mathrm{~g} / 100 \mathrm{~g}$, normal protein content and $10 \%$ fat), (2) moderate MLPD ( $9 \mathrm{~g} / 100 \mathrm{~g}, 50 \%$ protein diet and $10 \%$ fat $)$ and (3) severe MLPD (6g/100 g, 33\% protein diet and 10\% fat) 1 week before 
mating (to avoid the stress of a food change). We replaced protein with carbohydrates so that the diets were iso-caloric; the fat content was kept constant at $10 \%$. The normal diet contained $18 \mathrm{~g}$ protein per $100 \mathrm{~g}$ food, as described by guidelines published by Nutrient Requirements of Laboratory Animals, Fourth Revised Edition, $1995 .{ }^{7}$ We measured the amount of food (grams per day) consumed by the mice on both the normal diet and the isocaloric low-protein diets (control, $9 \mathrm{~g}$ and $6 \mathrm{~g}$ ). The amount of food (grams per day) consumed by the mice on the normal diet and the mice on the isocaloric low-protein diets was similar: $3.48 \pm 0.54 \mathrm{~g}$ for females $(n=16)$ and $4.24 \pm 0.33 \mathrm{~g}$ for males $(n=16)$ in the control group, $3.37 \pm 0.41(n=16)$ for females and $4.12 \pm 0.34 \mathrm{~g}$ for males $(n=16)$ in the moderate MLPD group, and $3.29 \pm 0.61 \mathrm{~g}$ for females $(n=16)$ and $4.23 \pm 0.73 \mathrm{~g}$ for males $(n=16)$ in the severe MLPD group.

\section{Blood pressure measurement}

In numerous studies, the tail-cuff method has been shown to measure the blood pressure non-invasively and accurately in mice and rats. ${ }^{8,9}$ We measured the blood pressure non-invasively each week by determining the tail blood volume, flow and pressure with a volume pressure recording sensor and an occlusion tail cuff (CODA System, Kent Scientific, Torrington, CT, USA). This system is significantly different from the plethysmographic-based, tail-cuff measurement system, which measures only systolic blood pressure. ${ }^{10}$ This noninvasive, highly accurate system is capable of measuring both the systolic and diastolic blood pressure simultaneously with the heart rate. ${ }^{11,12}$ Before beginning the study, mice were trained for three 15 -min sessions each day for 3 days, or until we obtained stable blood pressure recordings.

\section{Blood glucose, serum insulin and leptin measurement}

Random blood glucose levels were measured by obtaining blood from the left ventricle at the time of euthanasia. The blood glucose was measured following the Jackson Laboratory Protocol. Briefly, $1 \mu \mathrm{l}$ of blood was applied on the test strip for Johnson and Johnson's OneTouch Ultra Blood Glucose Monitoring System (http://jaxservices.jax.org/glucosetolerance_protocol.html). Calibration of the OneTouch Ultra Blood Glucose Monitoring System was performed using control test solutions provided by the manufacturer (Lifescan, Milpitas, CA, USA). Serum insulin and leptin levels were measured utilizing commercially available Elisa Kits (Alpco Distributors, Salem, NH, USA), according to the manufacturer's instructions.

\section{Statistics}

We analyzed the data using repeated measure (RM), one-way and two-way Univariate Analysis of Variance (ANOVA) and Multivariate Analysis of Variance (MANOVA), with Bonferroni's and Tukey's post-hoc analysis as well as linear and non-linear regression, to determine the statistically significant differences between groups using GraphPad Prism software (GraphPad Software, San Diego, CA, USA) and IBM SPSS (Statistical Package for the Social Sciences) (IBM Corp., Armonk, NY, USA). The hypothesis was accepted at $P<0.05$. For the measurement of blood pressure, glucose, insulin and leptin in each study group, one male or female offspring, considered as $n=1$, was included from one mouse dam.

\section{RESULTS}

\section{Maternal protein deprivation and offspring weights}

Maternal protein restriction was associated with a significant reduction in birthweight: $1.47 \pm 0.03 \mathrm{~g}(n=20), 1.29 \pm 0.03 \mathrm{~g}(n=24)$ and $1.19 \pm 0.02 \mathrm{~g}(n=38)$ for the control, moderate and severe MLPD groups, respectively $(P<0.05$, by one-way ANOVA) (Figure 1a). Placental weights and litter size did not differ significantly (data not shown). These results demonstrate that a protein-reduced, iso-caloric diet can cause a dose-dependent intra-uterine growth restriction (IUGR) in mice.

Furthermore, there was rapid catch-up growth in the offspring from the protein-deprived groups. Irrespective of the maternal dietary group, the RM two-way ANOVA with linear and non-linear regression demonstrated a significant increase in the offspring's weight with age. By 4 weeks after birth, the mean weight was significantly higher in the severe MLPD group compared with the control and moderate MLPD groups (Figure 1a). A sexually dimorphic trend of catch-up growth was observed in males and females, as indicated by the significant interaction of sex $\times$ diet by two-way RM-ANOVA. Following rapid catch-up growth, males from the severe MLPD group remained significantly overweight throughout their lives compared with the control and moderate MLPD groups (Table 1 and Figure 1c). However, females from the control group weighed more than those from the severe MLPD group (Table 1 and Figure 1b). Notably, females in the moderate MLPD group had a significantly greater catch-up growth than those of the control group (Table 1 and Figure $1 \mathrm{~b}$ ). We also recorded body and organ weights when the mice were euthanized at 32 weeks of age. Table 1 shows the body weight and the organ weights as a percent of the body weight (\%BW) at 32 weeks of age. Using MANOVA, only the kidney, of all the organs examined, had a significantly lower $\% \mathrm{BW}$ in the control female mice compared with the control male mice. Moreover, there was a significant reduction in the brain \%BW in both males and females of the moderate MLPD group, whereas in the severe MLPD group there was a significant reduction in brain \%BW only in males. We also observed a significant decrease in the lung and kidney \%BW in females from the moderate MLPD group compared with the females from the control group.

\section{Maternal protein deprivation and blood pressure in offspring}

As shown in Figures 2 and 3, female mice have significantly higher blood pressure than their male counterparts, and a significant interaction of sex $\times$ diet was observed with an RM two-way ANOVA by 16th, 16th and 14th week of age for mean arterial, systolic and diastolic blood pressure, respectively. The sex $\times$ diet interaction remained significant until the latest blood pressure measurement was conducted at 24 weeks of age. Moreover, maternal protein restriction was associated with increased systolic, diastolic and mean blood pressure in mouse offspring in both the moderate and the severe MLPD groups in both sexes. However, the increase was more pronounced in the female offspring (Figures 2a, b and 3) compared with the male offspring (Figures $2 \mathrm{c}-\mathrm{f}$ and $3 \mathrm{~b}$ ). Using RM-ANOVA, in females from the control, moderate and severe MLPD groups, the increase in blood pressure was observed at approximately 10 weeks of age, whereas in males from both MLPD groups, the increase in blood pressure was observed at approximately 15 weeks of age. As a consequence of moderate and severe antenatal MLPD, we observed with RM-ANOVA a significant increase in the mean, systolic and diastolic arterial blood pressure beginning starting at the 16th, 16th and 14th week of age, respectively, in the female offspring compared with their male counterparts.

\section{Maternal protein deprivation and heart rates in offspring} As shown in Figure 4c, males had a significantly higher HR compared with females. In males, we determined with RM-ANOVA a significant increase in $\mathrm{HR}$ as a consequence of both moderate and severe antenatal MLPD administration (Figure $4 \mathrm{~b}$ ). In contrast, no significant association was observed between different diets and sex on HR in females (Figure 4a). Importantly, a significant interaction of sex $\times$ diet was observed by two-way ANOVA on HR as a consequence of antenatal MLPD. 


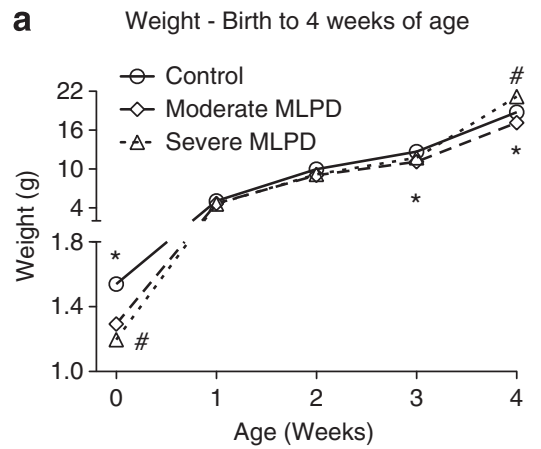

$(\mathrm{N}=20,24$, and 38 in control, 50\% MLPD, and $33 \%$ MLPD group, respectively.

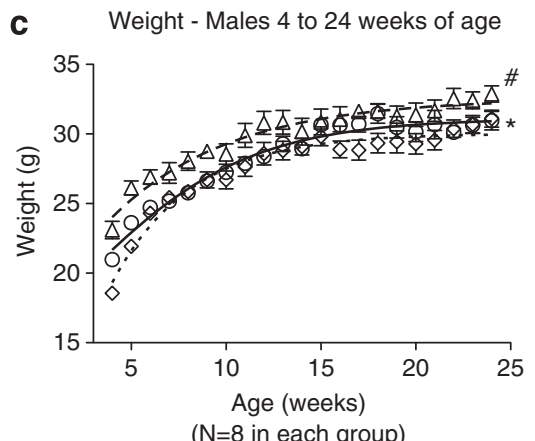

b Weight - Females 4 to 24 weeks of age

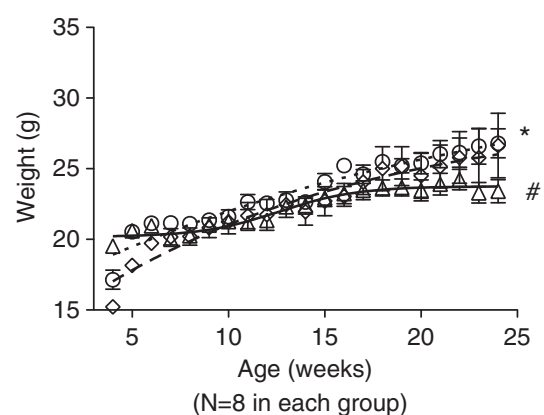

d Weight - Females Vs Males

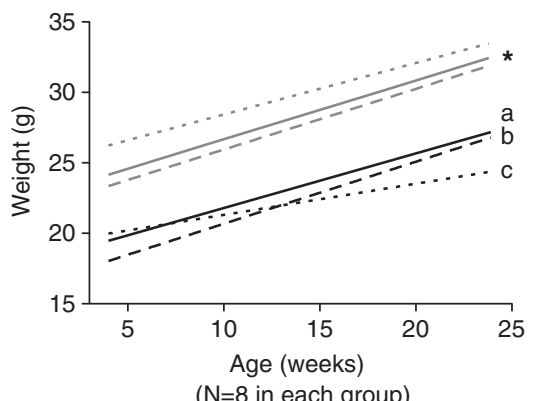

Figure 1 Offspring weight from control and maternal low-protein diet (MLPD) groups. (a) Offspring weight from birth until 4 weeks of age. * denotes significant difference $(P<0.05)$ between control and moderate MLPD groups at birth, 3 weeks and 4 weeks of age, whereas \# denotes significant difference $(P<0.05)$ between control and severe MLPD groups at birth and 4 weeks of age by one-way analysis of variance (ANOVA). (b) Female offspring weight from 4 weeks until 24 weeks of age. (c) Male offspring weight from 4 weeks until 24 weeks of age. Circle with solid black line, diamond with stippled black line and triangle with dotted black line denotes control, moderate MLPD and severe MLPD groups, respectively. For (b) and (c) * denotes significant difference $(P<0.05)$ between control group and moderate MLPD group, whereas \# denotes significant difference $(P<0.05)$ between control and severe MLPD groups by repeated measure (RM) two-way ANOVA with diet and sex as main factors. (d) Comparison of rapid catch-up growth in male and female offspring by RM two-way ANOVA and multiple linear regression analysis. Black lines denotes female and gray lines denotes male groups. Solid lines, stippled lines and dotted lines denote control, moderate MLPD and severe MLPD groups, respectively. $a, b$ and $c$ denote significant difference $(P<0.05)$ between male and female offsprings, and * denotes a significant interaction between sex $\times$ diet on body weight as determined by RM two-way ANOVA with sex and diet as main factors.

Table 1 Shows mean body and organ weight expressed as \% of body weight with \pm s.e. of mean at 32 weeks of age

\begin{tabular}{|c|c|c|c|c|c|c|}
\hline & \multicolumn{2}{|c|}{ Control } & \multicolumn{2}{|c|}{ Moderate MLPD } & \multicolumn{2}{|c|}{ Severe MLPD } \\
\hline & Males & Females & Males & Females & Males & Females \\
\hline Brain & $1.87 \pm 0.07(7)$ & $2.04 \pm 0.06(7)$ & $1.58 \pm 0.063(8)^{\mathrm{a}}$ & $1.67 \pm 0.11(5)^{\mathrm{a}}$ & $1.55 \pm 0.05(6)^{\mathrm{a}}$ & $2.1 \pm 0.11(7)$ \\
\hline Heart & $0.55 \pm 0.034(7)$ & $0.506 \pm 0.039(7)$ & $0.519 \pm 0.019(8)$ & $0.451 \pm 0.028$ & $0.529 \pm 0.014(6)$ & $0.58 \pm 0.044$ \\
\hline Lung & $0.647 \pm 0.044(7)$ & $0.666 \pm 0.018(7)$ & $0.590 \pm 0.026(8)$ & $0.536 \pm 0.043(5)^{a}$ & $0.580 \pm 0.020(6)$ & $0.712 \pm 0.023(7)$ \\
\hline Adrenals & $0.0888 \pm 0.017(7)$ & $0.0982 \pm 0.011(7)$ & $0.0736 \pm 0.008(8)$ & $0.0993 \pm 0.005$ (5) & $0.0605 \pm 0.003(6)$ & $0.0972 \pm 0.015(7)$ \\
\hline Pancreas & $0.534 \pm 0.025$ & $0.561 \pm 0.028$ & $0.495 \pm 0.023$ & $0.494 \pm 0.041$ & $0.527 \pm 0.033(6)$ & $0.746 \pm 0.095$ \\
\hline
\end{tabular}

Abbreviation: MLPD, maternal low-protein diet.

Numbers in parentheses represent the number of mice.

aDenotes significant difference between control and moderate or severe MLPD groups.

Maternal protein deprivation and blood glucose, serum insulin and leptin levels

As shown in Figure 5a, whereas the blood glucose levels were significantly increased in females from the moderate MLPD group compared with the control and severe MLPD groups, we observed no change in blood glucose levels in the male offspring from any group.
Additionally, serum insulin levels did not differ among any of the study groups (Figure $5 b$ ). As shown in Figure $5 c$, the serum leptin levels in the control females were significantly higher than those in the control males. Moreover, serum leptin levels remained elevated in females in the moderate MLPD group but were significantly lower in the severe MLPD group compared with the females in the control 

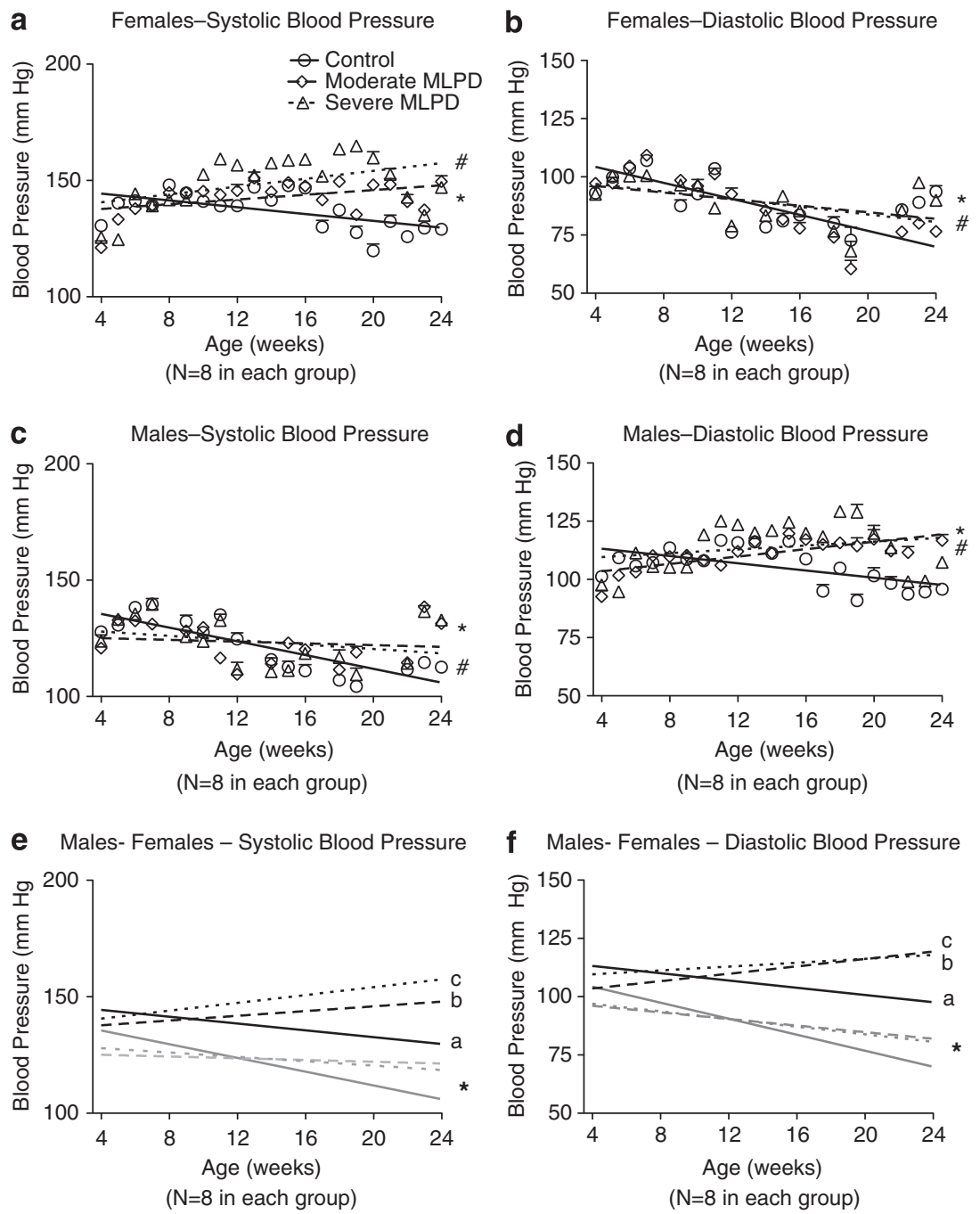

Figure 2 Offspring systolic and diastolic blood pressure from 4 weeks until 24 weeks as a consequence of maternal low-protein diet (MLPD). (a) Systolic blood pressure of female mice. (b) Diastolic blood pressure of female mice. (c) Systolic blood pressure of male mice. (d) Diastolic blood pressure of male mice. * denotes significant difference $(P<0.05)$ between control and moderate MLPD groups for diet and sex as main factors by repeated measure $(\mathrm{RM})$ two-way analysis of variance (ANOVA). \# denotes significant difference $(P<0.05)$ between control and severe groups for diet and sex as main factors by RM two-way ANOVA. (e) and (f) show comparison of males and females systolic and diastolic blood pressures in three diet group as analyzed by multiple regression and RM two-way ANOVA. Black lines denote female and gray lines denote male groups. Solid lines, stippled lines and dotted lines denotes control, moderate MLPD and severe MLPD groups, respectively. a, b and c denotes significant difference $(P<0.05)$ between male and female subjects, and * denotes a significant interaction between sex $\times$ diet on systolic (e) and diastolic (f) as determined by RM two-way ANOVA with sex and diet as main factors.

group. In males, serum leptin levels did not differ significantly (Figure 5c). In addition, we also observed a negative correlation between the body weight and leptin levels in both males and females from the moderate MLPD group (data not shown).

\section{DISCUSSION}

In this study, we report the sexually dimorphic programming of hypertension and overweight in adult mice offspring as a consequence of an antenatal MLPD. As noted, a considerable body of epidemiological data in humans has demonstrated that the developmental programming of hypertension may occur as a consequence of dietary manipulations during pregnancy. ${ }^{1,13,14}$ In addition, experiments in several species of laboratory animals have demonstrated the importance of maternal nutrition during gestation in the genesis of hypertension in their adult offspring, including rats, ${ }^{5}$ guinea pigs ${ }^{15}$ and sheep. ${ }^{4}$

Notably, this study demonstrates differential programming in male and female offspring in response to both moderate and severe protein restriction during gestational life (Figures 2 and 3). In the female offspring of both the moderate and severe MLPD groups, the development of hypertension began early (approximately 10 weeks of age). In contrast, the increased blood pressure in the males in the MLPD groups was observed significantly later (approximately 15 weeks of age) and was not as pronounced as that in females. This study demonstrates that in $\mathrm{FVB} / \mathrm{NJ}$ mice, the female sex is more susceptible to the developmental programming of hypertension as a consequence of antenatal MLPD. Similar findings of an increase in the arterial pressure in adulthood (sexes not differentiated), with the 
Females - Mean Arterial Blood Pressure

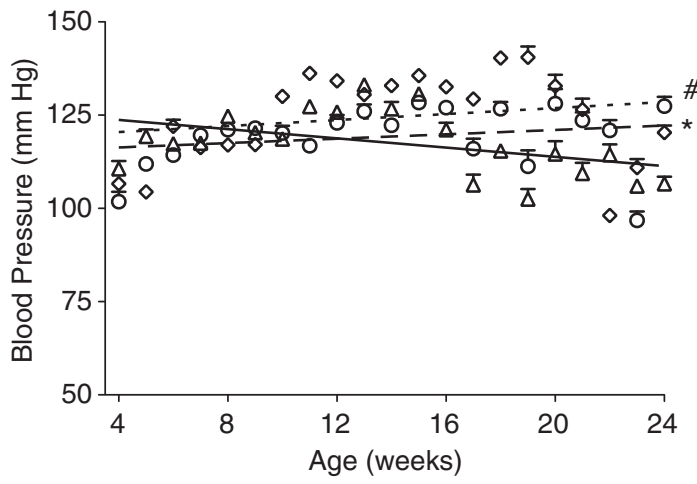

$(\mathrm{N}=8$ in each group)

b

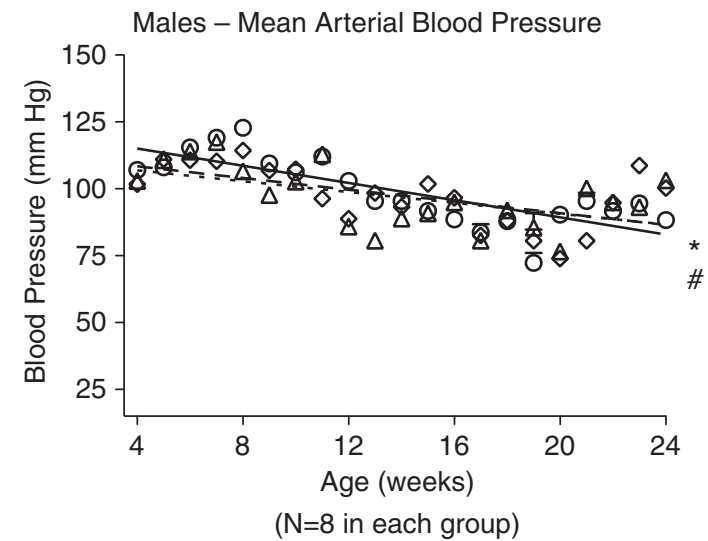

C Males - Females - Mean Arterial Blood Pressure

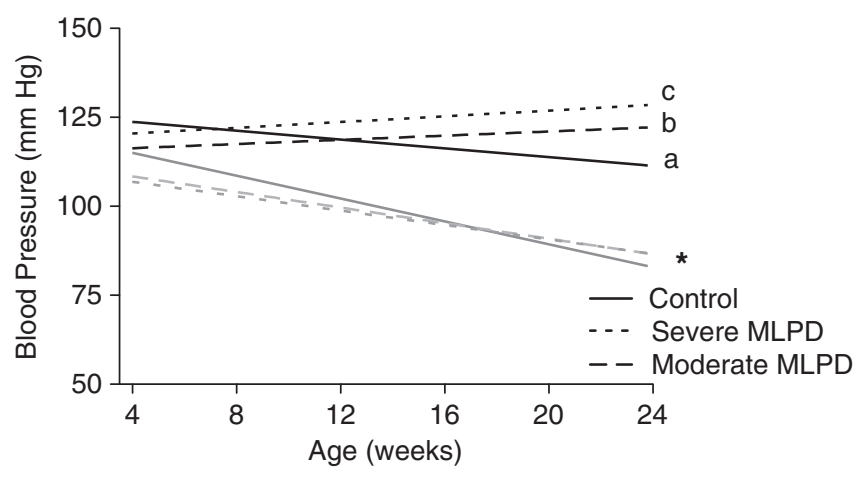

( $\mathrm{N}=8$ in each group)

Figure 3 Mean arterial blood pressure from 4 weeks until 24 week of age as a consequence of maternal low-protein diet (MLPD). (a) Mean arterial blood pressure in female mice offspring. (b) Mean arterial blood pressure in male mice offspring. Circle with black line, diamond with stippled line and triangle with dotted line denotes control, moderate, and severe MLPD groups. * denotes significant difference $(P<0.05)$ between control and moderate MLPD groups for diet and sex as main factors by repeated measure (RM) two-way analysis of variance (ANOVA). \# denotes significant difference $(P<0.05)$ between control and severe groups for diet and sex as main factors by RM two-way ANOVA. (c) Shows comparison of males and females mean arterial blood pressure. Black lines denote female and gray lines denote male groups. Solid lines, stippled lines and dotted lines denotes control, moderate MLPD and severe MLPD groups, respectively. a, $\mathrm{b}$ and $\mathrm{c}$ denote significant difference $(P<0.05)$ between male and female subjects, and * denotes a significant interaction between sex $\times$ diet on mean arterial blood pressure as determined by RM two-way ANOVA with sex as a main factor and diet as a second factor. a

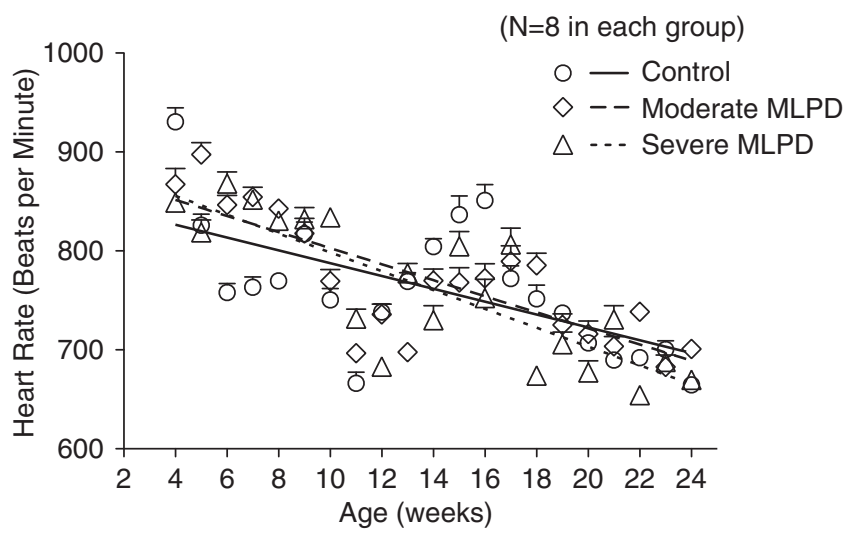

Males - Heart Rate

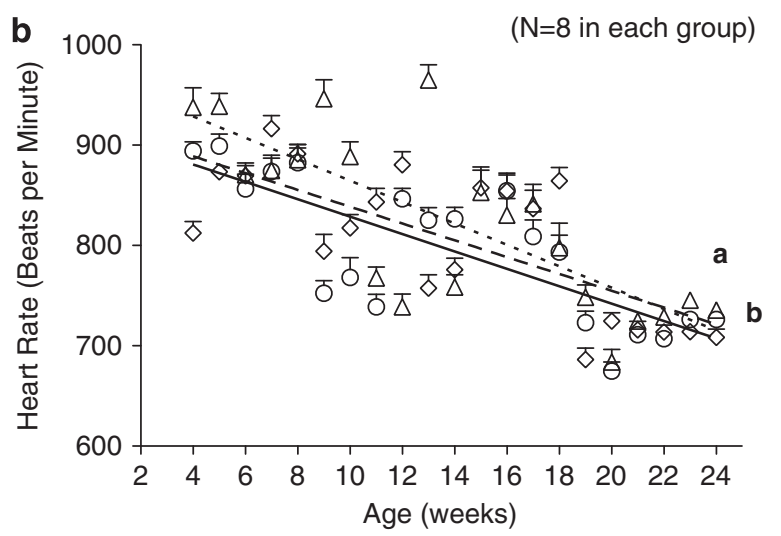

Male - Females - Heart Rate

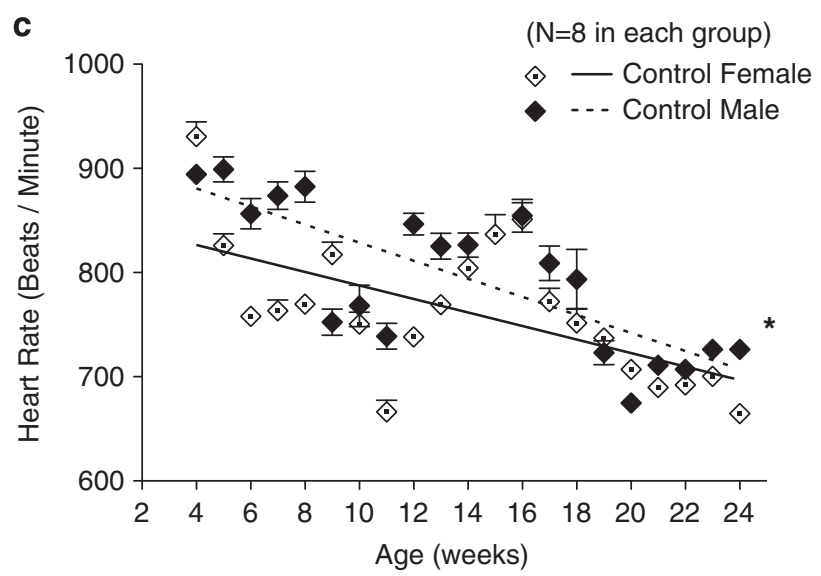

Figure 4 Offspring heart rate from 4 weeks until 24 weeks of age as a consequence of maternal low-protein diet (MLPD). (a) Heart rate in female offspring. (b) Heart rate in male offspring. Circle with black line, diamond with stippled line and triangle with dotted line denotes control, moderate MLPD and severe MLPD groups, respectively. a denotes significant difference $(P<0.05)$ between control and moderate MLPD groups for diet and sex as main factor by repeated measure (RM) two-way analysis of variance (ANOVA). b denotes significant difference $(P<0.05)$ between control and severe groups for diet as a main factor by RM two-way ANOVA (c) Male and female heart rates. * denotes significant difference between male and female subjects and sex $\times$ diet interaction on heart rate as determined by RM two-way ANOVA with sex and diet as main factors. 
a

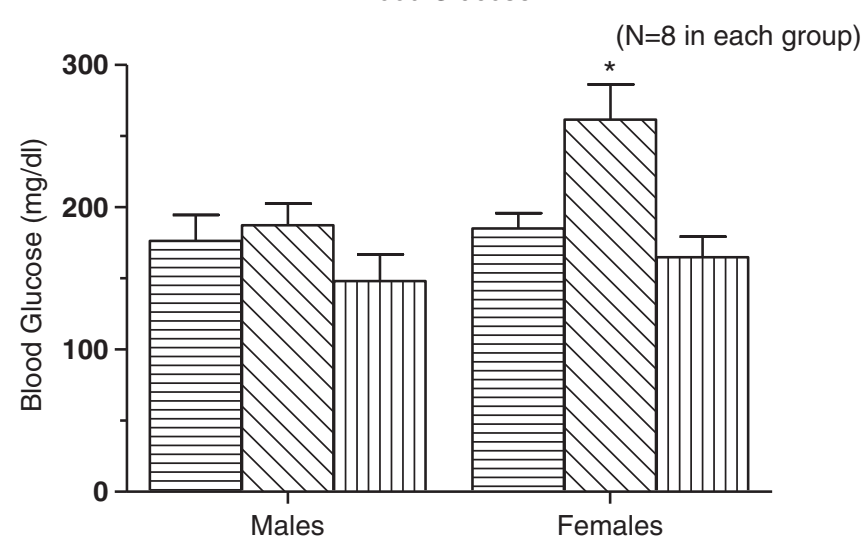

b

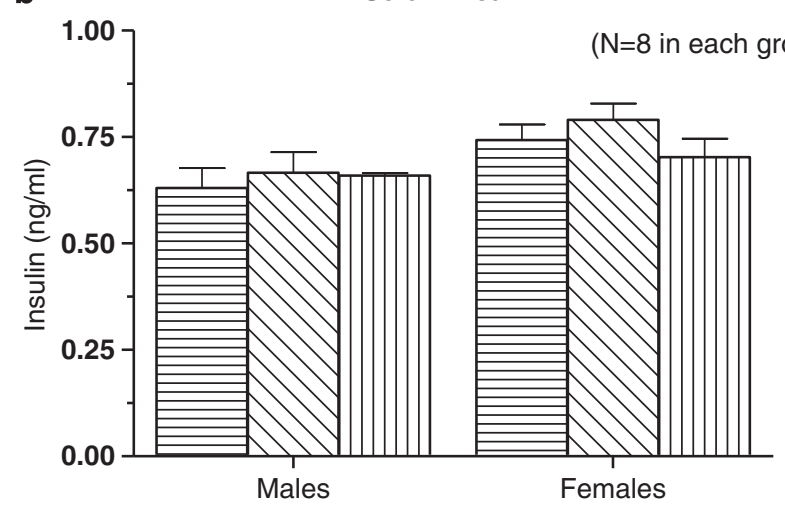

C

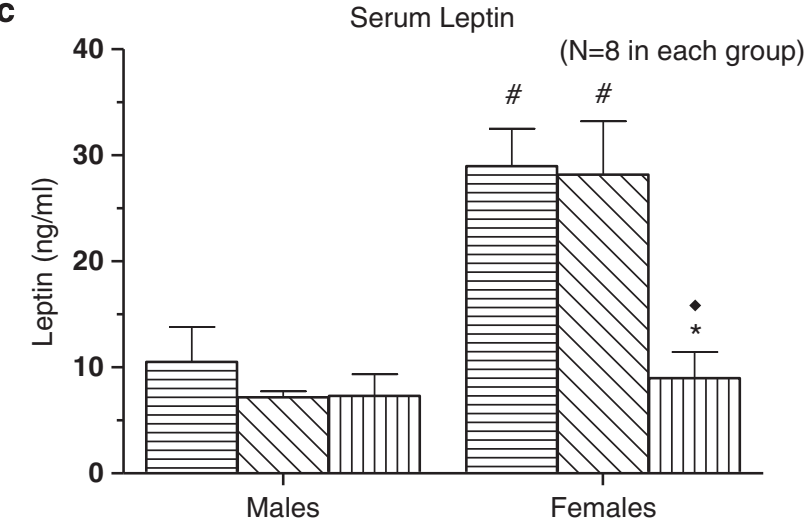

Figure 5 Bar graphs showing offspring glucose (a), serum insulin (b) and leptin (c) levels at 32 weeks of age in all six groups. Bars with horizontal, angled and vertical stripes represent control, moderate and severe maternal low-protein diet (MLPD) groups, respectively. * denotes significant difference $(P<0.05)$ between control and MLPD groups. \# denotes significant difference $(P<0.05)$ between males and females of same group. - denotes significant difference $(P<0.05)$ between moderate and severe MLPD groups.

effects being greater with more severe restrictions, have been observed in other species. ${ }^{16-18}$

Another important finding of this study was the increased blood glucose levels in females in the moderate, but not the severe, MLPD group (Figure 5a). Moreover, in this strain of mice, we did not observe increased blood glucose in males from either the moderate or severe MLPD group. We also did not observe any maternal diet-associated differences in serum insulin levels that would suggest insulin resistance (Figure 5b). Nonetheless, maternal protein restriction and global malnutrition have been linked to an increased incidence of Type 2 diabetes in humans ${ }^{19-21}$ and in several species of laboratory animals. ${ }^{3,22}$ Several reasons may account for our observations in the present study. One may be that these changes are specific to this particular mouse strain. Another is that changes in dietary components are known to differentially program physiological changes in humans and laboratory animals. ${ }^{3}$ Also of potential importance, the present study demonstrated significantly higher (threefold) basal leptin levels in females compared with males (Figure 5c). Of importance, similar to our findings, evidence in humans supports that females have significantly higher leptin levels compared with males. ${ }^{23}$ In response to MLPD, we observed significantly decreased serum leptin levels only in the severe MLPD group. Overall, the findings indicate the differential programming of diabetes and hypertension as a consequence of a MLPD in a dose-dependent manner.

In this study, we also observed a significant reduction in birthweight, which increased with the severity of protein deprivation (Figure 1a). Thus, despite an iso-caloric diet with adequate minerals and vitamins, these mice delivered IUGR pups. This finding supports the importance of a balanced diet in humans for the prevention of IUGR. Several studies in humans and laboratory animals indicate IUGR as a risk factor for hypertension, Type 2 diabetes and other diseases. ${ }^{24,25}$ Moreover, in the present study, we also observed rapid catch-up growth, which was more pronounced in the severe MLPD group. Rapid catch-up growth has been linked to hypertension in several studies ${ }^{26,27}$ and was also observed in the present study. In both males and females, serum leptin levels correlated inversely with increasing body weight in both control and severe MLPD groups. Additionally, the leptin levels were reduced significantly in the female offspring from the severe MLPD groups. Leptin is known to act on the hypothalamus to inhibit appetite, which suggests that the female offspring from the severe MLPD group were programmed to have an increased appetite (lower leptin). Our findings concur with a recent report on rats that also showed a decrease in leptin with food restriction. ${ }^{28}$ Other studies in humans link a low birthweight and rapid catch-up growth to the development of obesity. ${ }^{1,29,30}$ Nonetheless, low birthweight and rapid catch-up growth are well-known risk factors for hypertension and cardiovascular diseases in several human epidemiological studies ${ }^{26,31}$ and is consistent with the findings of the present study.

Overall, the present study demonstrates that in FVB/NJ mice, MLPD during gestation produces low birthweights, rapid catch-up growth and hypertension in the offspring. In females, moderate MLPD was also associated with increased blood glucose, whereas severe MLPD was associated with a significant decrease in plasma leptin.

\section{Perspective}

The findings of this study are consistent with the 'developmental origin of adult disease' and the 'thrifty phenotype' hypotheses. These hypotheses suggest that malnutrition of the fetus in utero may induce physiological and/or metabolic adaptations to ensure nutrient supply to the most vital organs at the expense of other organs. Such adaptations during critical periods may permanently reset or 'program' the metabolic system, which increases the fetus's chance of survival in a poor nutritional environment. Importantly, this may result in difficulty in coping with nutritional abundance in later life, with deleterious long-term consequences. This study provides evidence that as in humans, rats, guinea pigs and sheep, fetal 
programming also occurs in mice, which are a valuable tool for studying the genetic mechanisms of these responses. In our previous reports on the same mouse strain, we have shown several important changes in offspring gene expression in the renin-angiotensin system and systemic components, ${ }^{32}$ as well as locally in the placenta, ${ }^{33}$ lung, ${ }^{34}$ and brain $^{35}$ as a consequence of maternal stress during pregnancy. Nonetheless, elucidating the subcellular and molecular mechanisms of such programming remains a challenge.

\section{CONFLICT OF INTEREST}

The authors declare no conflict of interest.

1 Curhan GC, Chertow GM, Willett WC, Spiegelman D, Colditz GA, Manson JE, Speizer FE, Stampfer MJ. Birth weight and adult hypertension and obesity in women. Circulation 1996; 94: 1310-1315.

2 Barker DJ, Osmond C, Forsen TJ, Kajantie E, Eriksson JG. Maternal and social origins of hypertension. Hypertension 2007; 50: 565-571.

3 Langley-Evans SC. Critical differences between two low protein diet protocols in the programming of hypertension in the rat. Int J Food Sci Nutr 2000; 51: 11-17.

4 Gilbert JS, Lang AL, Grant AR, Nijland MJ. Maternal nutrient restriction in sheep: hypertension and decreased nephron number in offspring at 9 months of age. J Physiol 2005; 565: 137-147.

5 Brawley L, Poston L, Hanson MA. Mechanisms underlying the programming of small artery dysfunction: review of the model using low protein diet in pregnancy in the rat. Arch Physiol Biochem 2003; 111: 23-35.

6 Barker DJ, Bagby SP. Developmental antecedents of cardiovascular disease: a historical perspective. J Am Soc Nephrol 2005; 16: 2537-2544.

7 National Academy Press. Nutrient Requirements of Laboratory Animals. National Academy Press, Washington, D.C, 1995.

8 Johns C, Gavras I, Handy DE, Salomao A, Gavras H. Models of experimental hypertension in mice. Hypertension 1996; 28: 1064-1069.

9 Huang BS, White RA, Ahmad M, Jeng AY, Leenen FH. Central infusion of aldosterone synthase inhibitor prevents sympathetic hyperactivity and hypertension by central $\mathrm{Na}+$ in Wistar rats. Am J Physiol Regul Integr Comp Physiol 2008; 295: R166-R172.

10 Whitesall SE, Hoff JB, Vollmer AP, D'Alecy LG. Comparison of simultaneous measurement of mouse systolic arterial blood pressure by radiotelemetry and tail-cuff methods. Am J Physiol Heart Circ Physiol 2004; 286: H2408-H2415.

11 Starr A, Graepel R, Keeble J, Schmidhuber S, Clark N, Grant A, Shah AM, Brain SD. A reactive oxygen species-mediated component in neurogenic vasodilatation. CardiovasC Res 2008; 78: 139-147.

12 Shue E, Carson-Walter E, Liu Y, Winans B, Ali Z, Chen J, Walter K. Plasmalemmal Vesicle Associated Protein-1 (PV-1) is a marker of blood-brain barrier disruption in rodent models. BMC Neurosci 2008; 9: 29.

13 Barker DJ. The origins of the developmental origins theory. J Intern Med 2007; 261: 412-417.

14 Huxley RR, Shiell AW, Law CM. The role of size at birth and postnatal catch-up growth in determining systolic blood pressure: a systematic review of the literature. J Hypertens 2000; 18: 815-831.
15 Persson E, Jansson T. Low birth weight is associated with elevated adult blood pressure in the chronically catheterized guinea-pig. Acta Physiol Scand 1992; 145: 195-196.

16 Langley SC, Jackson AA. Increased systolic blood pressure in adult rats induced by fetal exposure to maternal low protein diets. Clin Sci 1994; 86: 217-222.

17 Vehaskari VM, Aviles DH, Manning J. Prenatal programming of adult hypertension in the rat. Kidney Int 2001; 59: 238-245.

18 Woods LL, Ingelfinger JR, Nyengaard JR, Rasch R. Maternal protein restriction suppresses the newborn renin-angiotensin system and programs adult hypertension in rats. Pediatr Res 2001; 49: 460-467.

19 Eriksson JG, Forsen T, Tuomilehto J, Jaddoe VW, Osmond C, Barker DJ. Effects of size at birth and childhood growth on the insulin resistance syndrome in elderly individuals. Diabetologia 2002; 45: 342-348.

20 Hales CN, Barker DJ. Type 2 (non-insulin-dependent) diabetes mellitus: the thrifty phenotype hypothesis. Diabetologia 1992; 35: 595-601.

21 Bhargava SK, Sachdev HS, Fall CH, Osmond C, Lakshmy R, Barker DJ, Biswas SK, Ramji S, Prabhakaran D, Reddy KS. Relation of serial changes in childhood body-mass index to impaired glucose tolerance in young adulthood. N Engl J Med 2004; 350: 865-875.

22 Kwong WY, Wild AE, Roberts P, Willis AC, Fleming TP. Maternal undernutrition during the preimplantation period of rat development causes blastocyst abnormalities and programming of postnatal hypertension. Development 2000; 127: 4195-4202.

23 Tatti P, Masselli L, Buonanno A, Di Mauro P, Strollo F. Leptin levels in diabetic and nondiabetic subjects. Endocrine 2001; 15: 305-308.

24 Thompson C, Syddall H, Rodin I, Osmond C, Barker DJ. Birth weight and the risk of depressive disorder in late life. Br J Psychiatry 2001; 179: 450-455.

25 Vickers $\mathrm{MH}$, Breier BH, Cutfield WS, Hofman PL, Gluckman PD. Fetal origins of hyperphagia, obesity, and hypertension and postnatal amplification by hypercaloric nutrition. Am J Physiol Endocrinol Metab 2000; 279: E83-E87.

26 Eriksson JG, Forsen TJ, Kajantie E, Osmond C, Barker DJ. Childhood growth and hypertension in later life. Hypertension 2007; 49: 1415-1421.

27 Huxley R. Does maternal nutrition in pregnancy and birth weight influence levels of CHD risk factors in adult life? Brit J Nutr 2004; 91: 459-468.

28 Takemori K, Kimura T, Shirasaka N, Inoue T, Masuno K, Ito H. Food restriction improves glucose and lipid metabolism through Sirt1 expression: a study using a new rat model with obesity and severe hypertension. Life Sci 2011; 88: 1088-1094.

29 Law CM, Barker DJ, Osmond C, Fall CH, Simmonds SJ. Early growth and abdominal fatness in adult life. J Epidemiol.Community Health 1992; 46: 184-186.

30 Ravelli AC, Van Der Meulen JH, Osmond C, Barker DJ, Bleker OP. Obesity at the age of $50 \mathrm{y}$ in men and women exposed to famine prenatally. Am J Clin Nutr 1999; 70: 811-816.

31 Barker DJ. Human growth and cardiovascular disease. Nestle Nutr Workshop Ser Pediatr Program 2008; 61: 21-38.

32 Goyal R, Galffy A, Field SA, Gheorghe CP, Mittal A, Longo LD. Maternal protein deprivation: changes in systemic renin-angiotensin system of the mouse fetus. Reprod Sci 2009; 16: 894-904.

33 Gheorghe CP, Goyal R, Holweger JD, Longo LD. Placental gene expression responses to maternal protein restriction in the mouse. Placenta 2009; 30: 411-417.

34 Goyal R, Leitzke A, Goyal D, Gheorghe CP, Longo LD. Antenatal maternal hypoxic stress: adaptations in fetal lung renin-angiotensin system. Reprod Sci 2011; 18: 180-189.

35 Goyal R, Goyal D, Leitzke A, Gheorghe CP, Longo LD. Brain renin-angiotensin system: fetal epigenetic programming by maternal protein restriction during pregnancy. Reprod Sci 2010; 17: 227-238. 